\title{
Mecanismo de Restauração em Rede Óptica WDM com Agregação de Tráfego
}

\author{
Eduardo J. Aloia, Amílcar C. César e Murilo A. Romero
}

\begin{abstract}
Resumo- Um algoritmo de restauração de tráfego nas camadas virtual e óptica é proposto neste artigo. $O$ algoritmo procura caminhos disjuntos pertencentes a um grupo de enlaces com risco compartilhado e utiliza agregação de tráfego na camada eletrônica da rede para reduzir a probabilidade de bloqueio de solicitações de conexão. A etapa de roteamento de tráfego e alocação de comprimento de onda é executada por meio de grafo. O desempenho do algoritmo é avaliado por meio de simulação computacional utilizando a topologia da rede óptica NSFNet. Os resultados numéricos mostram o desempenho do algoritmo utilizando as duas camadas de rede, de forma isolada ou simultaneamente, evidenciando a versatilidade e aplicabilidade da abordagem.
\end{abstract}

Index Terms-WDM; rede óptica; agregação de tráfego; falha; GMPLS; grupo de enlace com risco compartilhado; otimização; teoria de grafo.

Abstract - We propose an algorithm for traffic restoration in the optical and virtual layers of a optical network. The algorithm searches for disjoint paths belonging to a shared risk link group and uses traffic grooming in the electronic layer to decrease the blocking probability of connection requests. The routing and wavelength assignment task is carried out by a graph. The performance of the algorithm is evaluated by computer simulation based on the topology of the NSFNet optical network. Numerical results show the performance of the algorithm using the two layers, alone or simultaneously, indicating the versatility and applicability of the approach.

Index Terms-WDM; optical network; traffic grooming; fault protection; GMPLS; shared risk link group; optimization; graph theory.

\section{INTRODUÇ̃̃O}

As redes de telecomunicações têm atraído grande número de usuários em virtude dos inúmeros serviços oferecidos que incluem voz, dados e multimídia. As aplicações de vídeo são as que mais têm exigido largura de faixa. As redes ópticas WDM (wavelength division multiplexing) têm canalizado

Eduardo J. Aloia, Amílcar C. César e Murilo A. Romero, eduardoal56@gmail.com, amilcar@sc.usp.br, murilo.romero@)usp.br, Universidade de São Paulo, Escola de Engenharia de São Carlos, Departamento de Engenharia Elétrica, Av. Trabalhador São-carlense, 400, 13566-590 São Carlos, SP. Esta pesquisa foi parcialmente financiada pelas agências Capes e CNPq. grande parte do tráfego, operando em taxas de bit de 2,5, 10 e 40 Gbps.

Sendo assim, enorme quantidade de tráfego de usuários está presente nos vários comprimentos de onda dos enlaces ópticos, exigindo da rede esquemas de proteção e restauração para garantir a sobrevivência das conexões em presença de falha. Em outras palavras, se uma fibra é rompida, desconectando um ou vários caminhos ópticos (ligthpaths), um caminho alternativo deve ser acionado para circundar a falha e restabelecer o tráfego interrompido.

Esquemas de proteção acionam recursos previamente reservados assim que uma falha é detectada, enquanto esquemas de restauração atuam depois que uma falha ocorre, procurando por recursos disponíveis para desviar o tráfego. Nos dois esquemas há o caminho principal (de trabalho) e o caminho secundário, que pode ou não ser utilizado no período de operação sem falha [1]-[3].

As redes de telecomunicações são constituídas por várias camadas, formando uma arquitetura de protocolos. Visando simplificar esta arquitetura para ampliar a eficiência, o IETF padronizou o plano de controle GMPLS (generalized multiprotocol label switching), que opera com base em troca de rótulos com significado local nos roteadores. Há duas abordagens para este plano de controle: o modelo de pares (peer) e o modelo coberto (overlay).

Para roteadores IP os rótulos designam principalmente portas de entrada e saída. Para redes ópticas os rótulos designam portas de entrada e saída e comprimentos de onda ou grupo de comprimentos de onda para cada OXC (optical crossconnect). Para dispositivos de multiplexação por divisão de tempo e equipamentos SONET/SDH, os rótulos designam janelas temporais (time slots) de entrada e saída.

Desta forma, temos duas camadas de rede sob o plano de controle GMPLS: a camada eletrônica, formada por roteadores IP (Internet protocol), e a camada física. As rotas são estabelecidas na camada eletrônica e o tráfego entre os roteadores de origem e destino é roteado por caminho óptico, formado por enlaces na camada física. Assim, uma rota virtual estabelecida na camada eletrônica é roteada na camada óptica.

Neste artigo descrevemos um algoritmo para encontrar caminhos disjuntos pertencentes a um grupo de enlaces com risco compartilhado (shared risk link group - SRLG). Um conjunto de enlaces constitui um grupo de enlaces com risco compartilhado se compartilham um recurso cuja falha pode afetar todos os enlaces do conjunto. 
Adicionalmente, para garantir uma utilização mais eficiente dos recursos da rede quando tráfego de baixa taxa de transmissão é alocado em um canal óptico, incluímos nas funcionalidades da camada eletrônica a agregação de tráfego.

O algoritmo utiliza dois métodos de restauração de conexões afetadas por falha. O primeiro método, restauração na camada física, procura um caminho alternativo somente na topologia física da rede, não sendo permitida agregação de tráfego. O segundo método, restauração nas camadas física e virtual, procura caminho alternativo nas topologias física e virtual. Neste caso, a agregação de tráfego é permitida porque ela é realizada na camada eletrônica via conversão eletrônicaóptica-eletrônica. Utilizamos o modelo de pares para gerenciamento, a topologia da rede NSFNet, o algoritmo Dijkstra para encontrar o caminho entre os nós origem e destino e o algoritmo first-fit para alocar comprimento de onda.

A contribuição desta pesquisa é a utilização de duas camadas de rede, a virtual e a física, combinada com agregação de tráfego para encontrar caminhos disjuntos pertencentes a um grupo de enlaces com risco compartilhado. A técnica permite aumentar a probabilidade de restauração da rede em caso de falha e reduzir a quantidade de solicitações de conexão bloqueadas.

Esta nossa abordagem é baseada em grafo proposto por Zhu e Mukherjee [4] e estende as funcionalidades do algoritmo proposto por $\mathrm{Xu}$ et. al. [3]. Em [4], os autores propõem algoritmos para várias políticas de agregação de tráfego com base em grafo, incluem duas camadas de rede, mas não abordam problemas de falhas. Em [3], os autores propõem algoritmo heurístico para resolver problemas de falhas em redes com base em grupo de enlaces com risco compartilhado, abordam apenas uma camada de rede e não incluem agregação de tráfego.

Este artigo está organizado da seguinte maneira. A Seção II descreve o plano de controle GMPLS. Na Seção III o grafo utilizado é discutido. Os mecanismos de recuperação de falhas e o algoritmo proposto são descritos na Seção IV. A Seção V descreve os resultados numéricos e a Seção VI, as conclusões.

\section{Modelo De Rede}

A topologia de rede utilizada é formada por duas camadas: camadas virtual e a física, como mostra a Figura 1.

Os roteadores IP/GMPLS que comutam rótulos (label switching router, LSRs) estão situados na topologia virtual e os OXC (optical crossconnect), que comutam comprimentos de onda, na topologia física. Um caminho óptico (lightpath) entre os nós fonte e destino é estabelecido por um ou mais enlaces de fibras ópticas, interconectando OXCs. Uma ligação entre dois LSRs nesta configuração não necessariamente corresponde a uma ligação direta entre os respectivos OXCs na topologia física.

Estas arquiteturas multicamadas podem ser gerenciadas separadamente - modelo coberto, ou conjuntamente - modelo de pares.
No caso do modelo coberto, tanto o roteamento dos caminhos ópticos sobre a topologia física quanto o roteamento das conexões sobre a topologia virtual são gerenciados por dois planos de controle. A camada cliente GMPLS [5], formada por roteadores LSRs, conhece somente os canais virtuais fornecidos pela topologia física e a estrutura interna da topologia física é invisível à camada cliente. No caso do modelo de pares, as duas camadas são gerenciadas por um plano de controle, que dispõe de todas as informações sobre as duas camadas. Desta forma, as decisões de roteamento dos canais ópticos e das conexões são tomadas de forma única.

Dependendo do modelo, existem duas abordagens para o problema de agregação de tráfego. Para o modelo coberto [6], a decisão de roteamento é independente nas duas camadas, pois cada uma delas tem seu próprio algoritmo de roteamento. Especificamente, o problema de estabelecimento de caminhos ópticos na topologia física é abordado como um problema de roteamento e alocação de comprimento de onda (routing and wavelength assignment - RWA). Para o modelo de pares [6] um algoritmo único de agregação de tráfego é necessário no plano de controle para fornecer caminhos ópticos e rotear o tráfego.

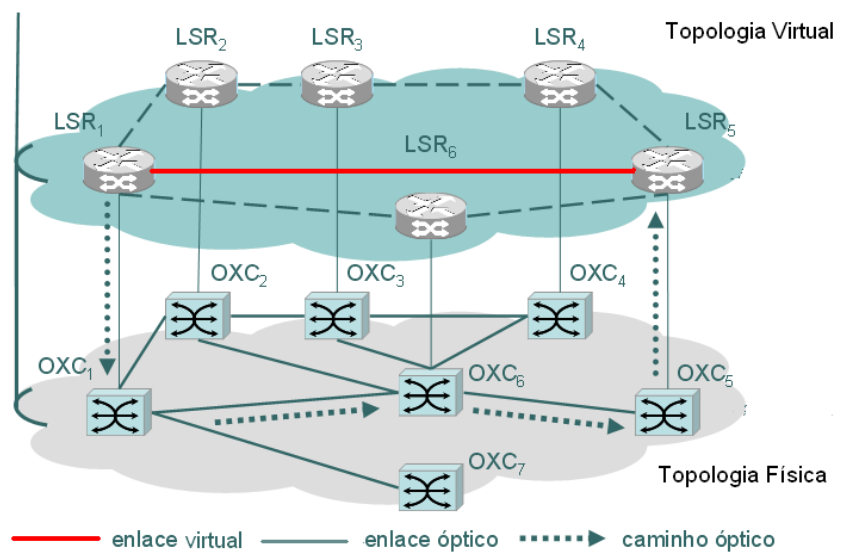

Figura 1. Topologia física e virtual em uma rede óptica.

Este artigo aborda a utilização de um grafo para o modelo de pares. O compartilhamento de informações sobre o estado da rede entre as duas camadas proporciona melhor uso dos recursos globais da rede, enquanto o modelo coberto leva à subutilização dos recursos [7].

\section{GRAFO UTILIZADO}

Para exemplificar o funcionamento do grafo [4], uma rede formada por três nós e dois comprimentos de onda é esquematizada na Figura 2. Seja o grafo $G(V, E)$ no qual $V$ é vértice e $E$ é aresta. Cada quadrado ou círculo com a letra "I" denota a porta de entrada em cada camada e o quadrado ou círculo com a letra "O" denota a porta de saída. O nó 3 está habilitado para conversão completa de comprimento de onda enquanto os demais não apresentam esta funcionalidade, evidenciada pela presença de arestas roxas (aresta de conversão, $\mathrm{AcC}$ ), que se cruzam entre as camadas do primeiro e segundo comprimento de onda. Os transmissores e 
receptores estão representados, respectivamente, pelas arestas AdT (aresta de transmissão) e AdR (aresta de recepção).

O grafo é composto por $W+2$ camadas, sendo $W$ o número de comprimentos de onda. As camadas 1 a $W$ denotam as camadas dos comprimentos de onda. É na camada do canal virtual que os enlaces entre os LSRs são configurados, ou seja, um enlace entre o nó 1 e 2 não apresentará necessariamente uma ligação na camada física (camada dos comprimentos de onda) exclusivamente entre os dois nós. Detalhes sobre a designação das arestas podem ser encontrados em [8], [9].

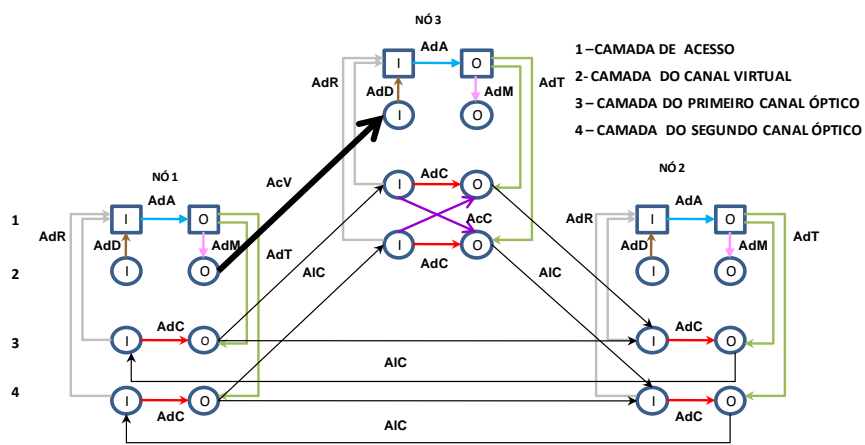

Figura 2. Grafo para uma rede com três nós e dois comprimentos de onda.

A política de agregação de tráfego utilizada é a minimização da rota na topologia física (MrTF), utilizada para rotear o tráfego pelos enlaces mais curtos na topologia física [9]. A Tabela 1 especifica os pesos alocados a cada aresta presente no grafo descrito acima.

TABELA 1. PESOS DESIGNADOS PARA A POLÍTICA DE AGREGAÇ̃̃O.

\begin{tabular}{|c|c|c|c|c|c|c|c|c|}
\hline Política & AlC & AdA & AdT & AdR & AcV & AdM & AdD & AdC \\
\hline MrTF & 1000 & 1 & 20 & 20 & X & 1 & 1 & 1 \\
\hline
\end{tabular}

Nota-se que o peso designado à aresta $\mathrm{AcV}$ para a política MrTF é X, sendo, portanto, passível de alteração, pois ele varia de acordo com a quantidade de enlaces ópticos utilizados para a configuração do caminho óptico.

\section{MECANISMOS DE RECUPERAÇÃO DE FALHAS}

No caso de restauração, os recursos são descobertos dinamicamente para a conexão interrompida. Em se tratando de proteção, os recursos são computados previamente e reservados [10], sendo mecanismo eficaz em caso de falha simples, como ruptura de fibra.

Os mecanismos de proteção tendem a ter um tempo de recuperação mais rápido, pois o caminho alternativo já é conhecido antes da ocorrência da falha. Já os mecanismos de restauração conhecem o estado da rede e tendem a ser mais eficientes na utilização dos recursos disponíveis (tais como enlaces ópticos) para o cálculo do caminho alternativo. Eles também podem ser utilizados para dotar as conexões de capacidade de sobrevivência sob condições de falhas múltiplas. Por outro lado, mecanismos de restauração não oferecem garantias de que haverá capacidade ociosa suficiente para recuperar as conexões afetadas.

O caminho principal ou de trabalho é a rota designada a uma conexão assim que for atendida e o caminho alternativo é a rota designada a uma conexão quando algum enlace pertencente ao seu caminho principal apresentar alguma falha.

Os esquemas de recuperação de falhas podem ser divididos em mecanismos de proteção e restauração de enlace, caminho e subcaminho. Estes mecanismos de proteção podem ser dedicados ou compartilhados. No mecanismo de proteção dedicado os recursos destinados aos caminhos alternativos (backup) não são compartilhados. Este mecanismo pode ser $1+1$, se o caminho principal e o alternativo transportam simultaneamente a mesma informação; ou 1:1, se somente o caminho alternativo transporta o tráfego se o caminho principal falhar. A vantagem do esquema 1+1 é o curtíssimo intervalo de tempo de recuperação. O esquema 1:1 é mais eficiente em capacidade, pois permite que os enlaces ópticos alocados para proteção sejam usados para o transporte de tráfego não-prioritário enquanto o caminho principal estiver operando sem falha. Em proteção compartilhada o recurso destinado a um caminho alternativo pode ser compartilhado por outro caminho alternativo, desde que os caminhos principais sejam disjuntos, para que não falhem ao mesmo tempo.

No exemplo particular em estudo aqui, a falha ocorre entre as conexões de número 50.000 e 60.000 , com probabilidade de ocorrência de $2 \%$. O esquema de restauração, cujo objetivo é encontrar um par de caminhos SRLG disjuntos, é baseado no seguinte algoritmo [9]:

\section{Alocar um canal óptico para a conexão;}

2. Verificar se a conexão solicitada está dentro da faixa de ocorrência de falha, entre as conexões 50.000 e 60.000; verificar se algum enlace da conexão falhou; classificar como conexão a ser restaurada;

\section{Eliminar do grafo os enlaces que falharam;}

4. Calcular o caminho SRLG disjunto. Se tal caminho existir ir para o passo 6;

5. Escrever 'não existe caminho de backup';

6. Fim.

O passo 4 deste algoritmo é implementado da seguinte maneira:

1. Após um caminho óptico (caminho principal) ser encontrado para uma determinada conexão e ser constatado que esta conexão necessita ser restaurada, um novo caminho SRLG-disjunto deve ser calculado. $O$ algoritmo elimina todos os enlaces que compõem o caminho principal e tenta encontrar um caminho de backup. Desta forma, todos os comprimentos de onda presentes nos enlaces utilizados pelo caminho principal são retirados do 
grafo. Caso não seja possível achar um caminho, significa que o caminho principal não possui um caminho de backup SRLG-disjunto, sendo necessário utilizar uma estratégia alternativa;

\section{Com este intuito, apenas os comprimentos de onda} utilizados pelo caminho principal são retirados do grafo e um novo caminho de backup é calculado. Agora, tais caminhos podem possuir enlaces comuns. $O$ vetor que armazena os enlaces comuns entre os caminhos principal e backup é o conjunto conflito-SRLG. Entenda-se por "enlaces comuns" não apenas os enlaces interligando os mesmos nós, mas também os que apresentam os mesmos SRLG.

3. Os enlaces presentes no conjunto conflito-SRLG são retirados do grafo. $O$ algoritmo tenta encontrar um novo caminho principal e um caminho backup SRLG-disjunto. $O$ algoritmo termina em duas situações: quando encontra um par caminho principal e caminho backup SRLG-disjunto; quando falha em encontrar um novo caminho principal ou um caminho de backup.

\section{RESUltados NumÉRICOS}

Nesta seção comparamos o desempenho de duas formas de restaurar tráfego dinâmico. A primeira considera apenas a topologia física (sem agregação de tráfego) e a segunda, a topologia física e a virtual (com agregação de tráfego). A rede utilizada na simulação é a NSFNet, mostrada na Figura 3. Escolhemos esta topologia por ser amplamente utilizada para avaliar desempenho de algoritmos RWA. O algoritmo proposto neste trabalho pode ser utilizado em qualquer topologia, como a da rede italiana de faixa larga ou da rede Ipê (rede nacional de ensino e pesquisa - RNP).

As solicitações de conexão seguem um processo de Poisson e são uniformemente distribuídos para todos os nós, sendo a rede submetida a uma carga de 60 erlangs. O tempo de permanência das conexões segue uma distribuição exponencial com tempo médio 60 s. A capacidade máxima de cada comprimento de onda é $10 \mathrm{Gbps}$. Cada solicitação de conexão pode dispor de taxa de transmissão de $m, 1 \leq m \leq g$, com $g=$ 4, cuja probabilidade de ocorrer é:

$$
R_{c}=\frac{1 / c}{\sum_{m=1}^{g} 1 / m},
$$

na qual $c$ pode ser $\{1,2,3,4\},\{1,1,1,1\}$ e $\{4,3,2,1\}$, respectivamente, para as conexões com taxa de transmissão 2,5 Gbps, 5,0 Gbps, 7,5 Gbps e 10 Gbps. As probabilidades calculadas por meio de (1) para os três conjuntos foram, respectivamente, $(48,24,16,12),(25,25,25,25)$ e $(12,16$, 24 , 48). Neste artigo utilizamos o primeiro conjunto de distribuição de probabilidade de geração de tráfego. Desta forma, são geradas solicitações com as seguintes probabilidades: $48 \%$ com taxa de transmissão $2,5 \mathrm{Gbps}, 24 \%$ com taxa de transmissão $5 \mathrm{Gbps}, 16 \%$ com taxa de transmissão 7,5 Gbps e $12 \%$ com taxa de transmissão 10 Gbps. Para a rede analisada cada enlace unidirecional de fibra admite 4 comprimentos de onda, todos os nós possuem capacidade de agregação e nenhuma capacidade de conversão de comprimento de onda. Cada nó possui transmissores e receptores suficientes para originar e terminar uma conexão, ou seja, o bloqueio de uma conexão não ocorrerá em virtude da falta de transmissores ou receptores em um nó, mas sim pela falta de caminhos ópticos. São geradas 100.000 conexões por meio da técnica de simulação por evento discreto. Para a alocação do comprimento de onda utilizamos o algoritmo first-fit.

As falhas ocorrem entre as solicitações de conexão 50.000 e 60.000 e a probabilidade de falha de um enlace da conexão é $2 \%$. A simulação foi realizada em um PC com processador Intel Core 2 de 2,4 GHz e 3,0 GB de memória RAM. O programa foi codificado em linguagem $\mathrm{C}$.

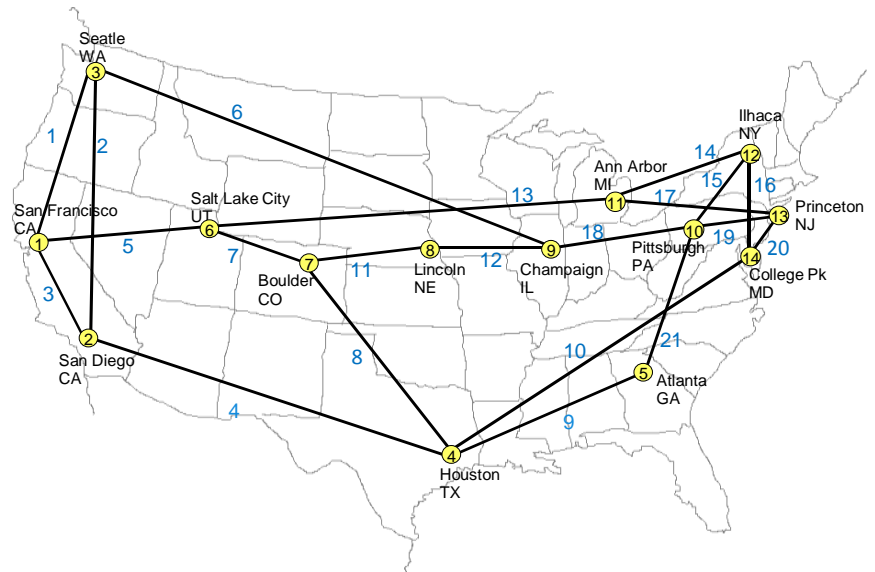

Figura 3. Rede NSFNet com os respectivos grupos de enlaces de risco compartilhado (SRLG) (em azul) alocados para cada enlace.

A Figura 4 apresenta a probabilidade de bloqueio para cada uma das 100.000 conexões quando são empregados os mecanismos de restauração na camada física e restauração nas camadas física e virtual. A espessura das linhas dos gráficos é causada pelo número elevado de pontos utilizados na confecção em sua confecção.

Quando a restauração é realizada apenas na camada física, a topologia virtual é ignorada, eliminando a possibilidade de agregar tráfego, possível apenas no domínio eletrônico. $\mathrm{Na}$ camada física, uma solicitação de conexão de $2,5 \mathrm{Gbps}$ é atendida apenas quando há comprimento de onda disponível. Se a capacidade máxima é $10 \mathrm{Gbps}$, o comprimento de onda alocado exclusivamente para a conexão de 2,5 Gbps fica com 7,5 Gbps "sem ocupação". Se não houver comprimento de onda disponível, a conexão é bloqueada. 


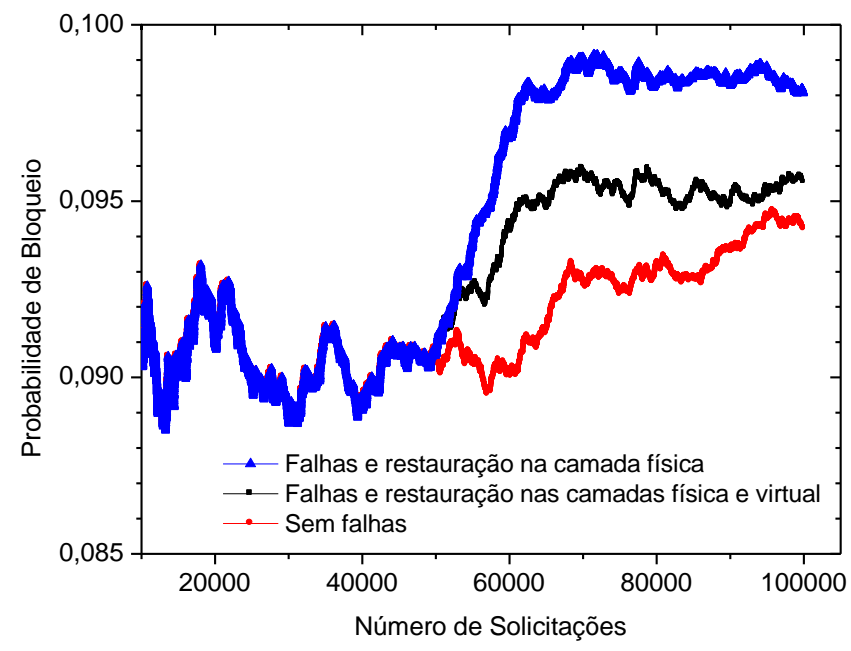

Figura 4. Probabilidade de bloqueio das conexões em operação sem falhas e com falhas e restauração somente na camada física e nas camadas física e virtual. As falhas ocorrem entre 50 e 60 mil solicitações.

Quando a camada virtual é utilizada, e com ela a possibilidade de agregar tráfego, aquela conexão de $2,5 \mathrm{Gbps}$ pode ser alocada em caminhos preexistentes, que já acomodam outras conexões. Assim, a agregação permite alocar número maior de conexões. Este comportamento pode ser corroborado pelos resultados mostrados na Figura 5, que apresenta a quantidade de conexões, com necessidade de restauração atendida, para os dois casos.

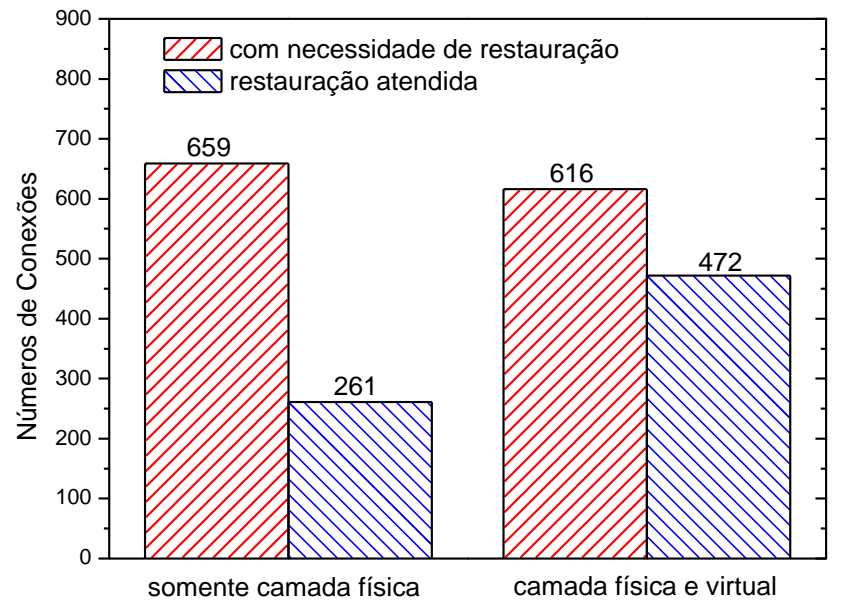

Figura 5. Número de conexões com necessidade de restauração e com restauração atendida somente com camada física e com camada física e virtual.

O número de conexões com necessidade de restauração e restauração atendidas para as 4 taxas de transmissão é mostrado nas Figuras 6 (somente camada física) e 7 (camada física e virtual, ou seja, com possibilidade de agregação de tráfego).

Observamos que a percentagem de conexões com a necessidade de restauração atendida vai diminuindo à medida que a taxa de transmissão aumenta. Embora a quantidade de conexões de 2,5 Gbps geradas seja maior, a agregação de tráfego as organiza e acomoda com eficiência. A capacidade máxima é $10 \mathrm{Gbps}$, acomodando 4 solicitações de 2,5 Gbps. Se um caminho já acomoda 2 solicitações de 2,5 Gbps e uma solicitação de 10 Gbps ocorre, esta não poderá ser alocada neste comprimento de onda, mas sim em um comprimento de onda exclusivo. Embora ocorram com probabilidades menores, à medida que a taxa aumenta será preciso comprimentos de onda com mais largura de banda óptica disponível.

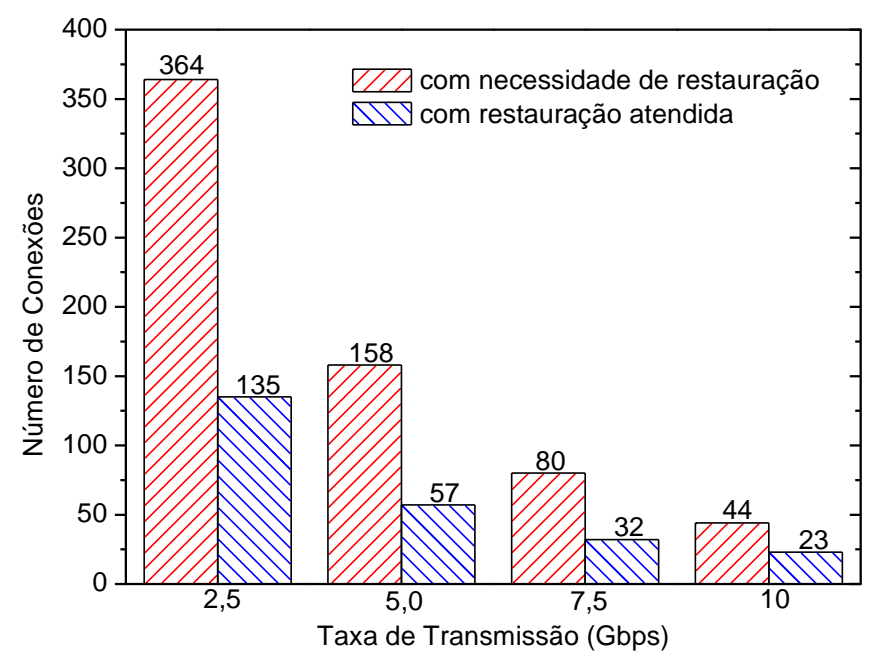

Figura 6. Número de conexões, distribuídas por largura de faixa, com necessidade de restauração e com restauração atendida somente na camada física (sem agregação de tráfego).

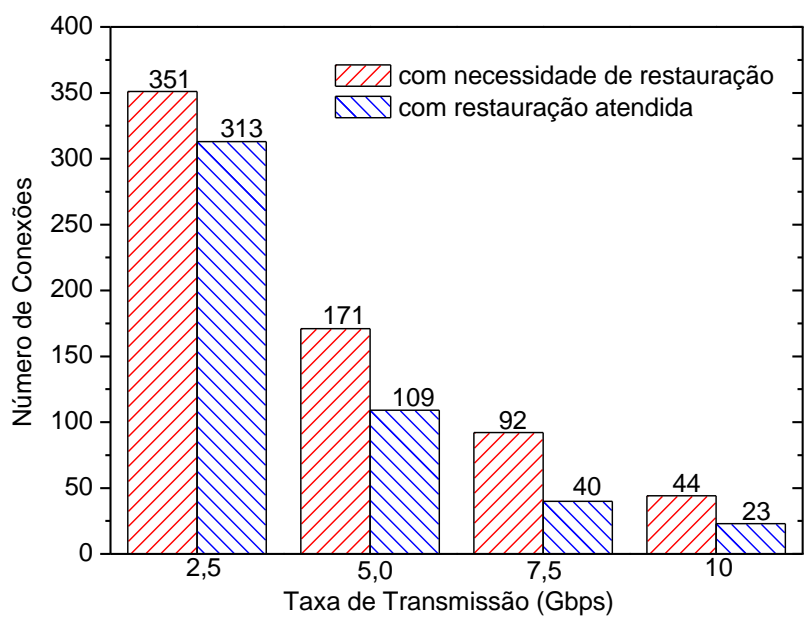

Figura 7. Número de conexões, distribuídas por largura de faixa, com necessidade de restauração e com restauração atendida na camada física (sem agregação de tráfego) e virtual (com agregação de tráfego).

Não apresentamos comparação direta com resultados obtidos em artigos de outros autores por causa da abordagem distinta. A abordagem deste trabalho também é heurística e a validação e conferência dos resultados são feitas com base em verificação de total de recursos, como quantidade de banda, comprimentos de onda e caminhos. 
Utilizamos o algoritmo first-fit para escolher comprimentos de onda disponíveis para alocar conexão. Este algoritmo é eficiente e sua implementação é simples. Entretanto, outras estratégias, como comprimento de onda mais (ou menos) utilizado, best-fit ou escolha aleatória poderiam ter sido adotadas. Na literatura há vários artigos comparando o desempenho destes algoritmos [11] e em simulações semelhantes à deste trabalho não foram verificadas diferenças significativas. Além disto, o foco da abordagem deste trabalho não é a técnica de alocação de comprimento de onda. Portanto, as comparações de desempenho foram realizadas na mesma base.

\section{CONCLUSÕES}

Um mecanismo de restauração de tráfego em rede óptica WDM com agregação de tráfego é proposto neste artigo. Utilizamos a topologia da rede NSFNet com os respectivos grupos de enlaces de risco compartilhado (SRLG) e quatro taxas de transmissão, geradas com probabilidades diferentes. Verificamos que o mecanismo de restauração utilizando simultaneamente a camada física e a virtual possibilitou atender número maior de conexões com necessidade de restauração do que utilizando restauração apenas na camada física. A técnica de agregação de tráfego, realizada na camada virtual, é responsável por esta melhoria.

A utilização da técnica de agregação de tráfego e mecanismo de restauração nas camadas física e virtual aumenta o atendimento de conexões com necessidade de restauração em relação ao mecanismo de restauração apenas na camada física.

$\mathrm{O}$ algoritmo proposto neste trabalho pode ser utilizado em qualquer topologia de rede. O grau dos nós da rede influi decisivamente nos resultados. Se uma topologia exibe nós com poucas ou apenas uma conexão, como é o caso da rede Ipê (RNP), então é de se esperar que a probabilidade de bloqueio seja elevada. Se um nó está conectado por apenas um enlace e ocorre uma falha, o nó ficará isolado da rede. Neste caso, um mecanismo de proteção, como duplicação de recursos, é mais adequado porque não haverá caminho alternativo para restauração. Quanto mais conectados forem os nós de uma topologia, menor será a probabilidade de bloqueio de uma conexão que necessite ser restaurada porque haverá mais possibilidade de se encontrar caminho alternativo. Por outro lado, fatores como carga de tráfego, taxa de geração das conexões e capacidade de agregar tráfego de baixas taxas de transmissão também influem nos resultados.

\section{REFERÊNCIAS}

[1] LI G.; Doverspike B.; Kalmanek C., "Fiber Span Failure Protection in Mesh Optical Networks", Optical Network Magazine, vol. 3, n 3, pp. 21-31, Maio/Junho 2002.

[2] Zhang J.; Mukherjee B., "A review of Fault Management in WDM Mesh Networks: Basic Concepts and Research Challenges". IEEE Network, pp. 41-48, Março/Abril 2004.

[3] Xu D; Xiong Y.; Qiao C., "Failure in Layered Networks with Shared Risk Link Groups”. IEEE Network, pp.36-41, Maio/Junho 2004.
[4] Zhu H.; Zang H.; R.; Mukherjee B., "A novel generic graph model for traffic grooming in heterogeneous WDM mesh networks". IEEE ACM Transactions on Networking, vol. 11, n 2, pp. 285 - 299, Abril 2003.

[5] E . Mannie at al, "Generalized Multi-Protocol Label Switching (GMPLS) Architecture". Internet Engineering Task Force. IETF RFC 3945, Outubro 2004. http://www.ietf.org.

[6] S. Koo, G. Sabin e S. Subramaniam, "Dynamic LSP provisioning in overlay, augmented, and peer architectures for IP/MPLS over WDM networks", Proc. of INFOCOM 2004, pp. 7-11, Hong Kong, China, Março 2004.

[7] E. Salvadori, L.R. Cigno e Z. Zsoka, "Dynamic grooming in IP networks base on the overlay architeture", Opt. Switch Network, vol. 3, Março de 2006, pp. 118-133.

[8] J.E. Aloia, A.C. César, M. A. Romero, "Agregação de tráfego e imparcialidade em redes ópticas WDM: Análise utilizando teoria de grafo"XXII Simpósio brasileiro de telecomunicações, Setembro 2007.

[9] J.E. Aloia, "Contribuições para a análise e simulação de redes ópticas". Tese de doutorado. EESC-USP, 140 p, 2009. http:// http://www.teses.usp.br/teses/disponiveis/18/18133/tde-14052009$162157 /$.

[10] A.A.D. Mello, "Suporte ao Tráfego Heterogêneo pela Rede Óptica: Habilidade de Sobrevivência". Tese de Doutorado. UNICAMP, 2006, $114 \mathrm{p}$.

[11] B. Mukherjee, Optical WDM Networks. Springer, pp.379-395, $1^{\text {a }}$ edição 2006. 